\title{
Digitizing Company Analytics - Digitalization Concept for Valuable Insights
}

\author{
J. Harkonen ${ }^{1}$, E. Mustonen ${ }^{1}$, J. Koskinen ${ }^{1}$, H. Hannila ${ }^{1}$ \\ ${ }^{1}$ Industrial Engineering and Management, University of Oulu, Oulu, Finland \\ (janne.harkonen@oulu.fi)
}

\begin{abstract}
Digitalizing business analytics by generating relevant information from data to gain valuable insights and to enhance decision-making is a key ability for companies. We describe a potential concept for digitizing company analytics to enable data-driven approach by providing a path from information needs to visualizing the analysis results, including the consideration of analysis logic, relevant data, and necessary data model. Company business processes, IT applications, and data assets provide the foundations to build upon. The developed concept may enable practitioners to consider possible applications and the needed, already existing technologies.
\end{abstract}

Keywords - Company analytics, digitalization, datadriven, data asset, data model, digitization, productization, product management.

\section{INTRODUCTION}

\section{A. Background}

Digitalization in business is challenged by old ways hindering new, and not understanding that it is not about technology [1], but about changing the way things are done. One of today's success factors involves digital business analytics, the ability of generating businessrelevant information from data to improve decisionmaking, business processes, products, services, and related activities [2]. This new ability is also linked to discussion on digital transformation [3], big data analytics [2], and industry 4.0 [4]. It is recognized how data and their exploitation can support the decision-making but are experienced challenging by companies [5]. The digital technologies and data change the approach on company activities [6], and data \& analytics provide a variety of application opportunities [7]. However, the new approach possibly requires a change in mindset and is experienced as challenging, and as effective business analytics can be a competitive advantage, the necessary concepts are not widely described.

Digitizing the approach to analytics by replacing the old analogue ways [8] of manual consolidation of data into spreadsheets [9] to exploit company data assets more systematically can prove beneficial by avoiding the challenges of the siloed enterprise application landscape [10]. Product, service, and portfolio management are examples of areas that can benefit of true digital analytics [8][11]. However, similarly markets [3][8], supply chain [2][7], processes [2], and other areas of business focus can benefit of the effective digital analytics. Further, digitalization can change the business by applying technology to solve problems and create value. Hence, digitalizing company ecosystems to enable true datadriven, real-time approach to business [12] can reduce the less factual aspects of decision-making. Initial basis for data-driven approach have been described, but examples are scarce, and discussion on viewpoints such as necessary data models, and technologies are lacking.

One of the more advanced examples working towards digital business analytics involves the active management of product portfolios over product lifecycles [13]-[14] that is complemented by an initial frame for product-centric data-driven decision-making [9]-[12][15], which applies productization concept and product structure to create consistency for data and to raise above company siloes and the challenges of system integration [10]-[11][16]. Business processes, enterprise applications, and data are focal to the foundations that are formed for data analytics [10]-[11][15]. These examples provide beneficial explanations up to the level of consistent data input, but not for other similar information needs, or further logic for obtaining information, related data models, or technologies that enable the analytics.

This study attempts to create a concept for digitizing company analytics to widen the perspective of data-driven approach and provide one possible description to support considering digital business analytics. The intention is to widen the understanding of possible targets of analysis by providing a path from information needs, considering the underlying analysis logic, and applying relevant data assets, to considering the data model and visualizing the analysis results.

\section{B. Methodology}

The study was carried out by reviewing the relevant literature and reflecting it against product portfolio analysis as an example of the application to demonstrate the logic. The methodology involves reviewing literature based on keyword searches and including relevant perspectives for further consideration. Specific interest was digital analytics, related logic, and data model with wider possibilities for analysis. A concept was constructed for digital data analytics by carefully considering the realities and the logic for data analytics. The initial validation of the concept involves discussions with industry experts and their feedback. The validation can be considered as a weak market test.

\section{RESULTS}

\section{A. Developed concept}




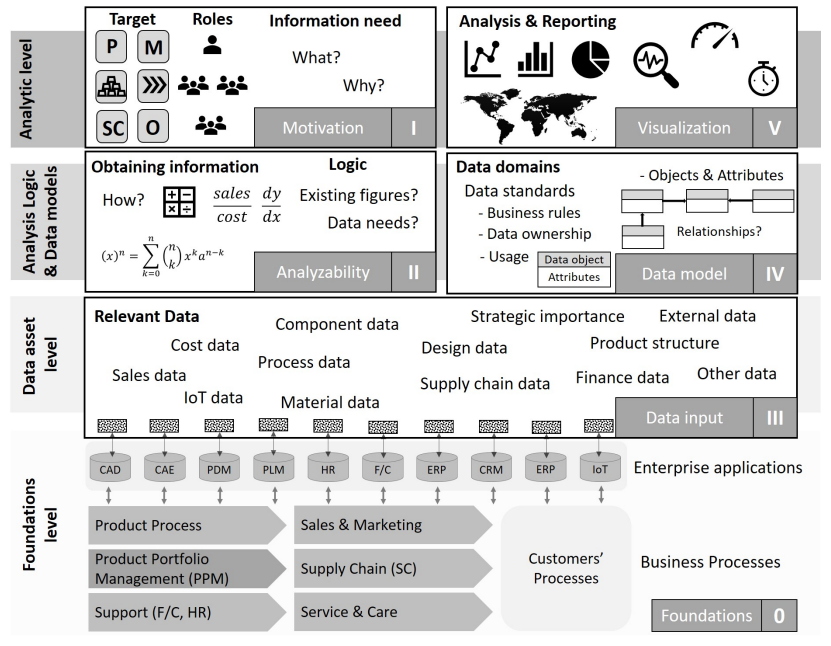

Fig. 1 Concept developed for company data analytics.

The developed concept (Figure 1) is an approach to company data analytics to consider the utilization of company data assets in a complex operating environment. The concept does not take a position on the technology to be utilized for the analytics. Nevertheless, the necessity of implementing a data model [11]-[12] is acknowledged. In this respect, considering product structure is one possibility to have a skeleton to support the data model [11]. This can be particularly beneficial especially for product and product portfolio related analysis. The product structure and productization can act as sources of consistency for the data [10].

The approach comprises four main levels and the related enablers. The top level, the "Analytic level", contains the considerations on analysis targets and motivations (Step I), based on which, the necessary data and analysis are visualized later to provide basis for reporting and decisions (Step V). The level also links to relevant roles. The second level, "Analysis logic \& Data models", contains the considerations on logic and needed data (Step II), and the data model involving data domains that include objects and attributes among other considerations (Step IV). These relate to the third "Data asset level", which involves the relevant data for the analytical needs (Step III). This third level links to the data assets that can be drawn from enterprise applications, likely the master data and transactions. Also, IoT data obtained via a variety of sensors can form a data asset. Variety of enterprise applications and external sources can be the sources of data assets. The enterprise applications, the IT, link to business processes, company internally, or those of customers. This way the business processes and the enterprise applications, and related data form the foundations for the concept (fourth "Foundations level"). Product master data, product data, company business processes and the enterprise applications provide a logical whole as the basis for analyses. The detailed description of the steps 0 through $\mathrm{V}$ follows.

\section{B. Foundations (0)}

The step zero involves the foundations. Business processes, the linking enterprise applications, and the related data form the foundations for data analytics [10][11]. The consistency of data is enabled by productization and product structure logic [10].

\section{Motivation (I)}

The first step of the developed concept focuses on the motivation for the analytics. The relevant questions involve the information needs, what information is needed, and why: Information needs also relate to corresponding roles, their tasks and goals, and may arise through different roles. The third aspect of motivation is the target of analysis: Targets of analysis can relate to Products (P), Markets (M), Product portfolio ( Process (m), Supply chain (SC), or other (O). Therefore, the aspects of motivation involve the information needs, roles, and targets of analysis.

For example, the role responsible for product portfolio management analysis, e.g. PPM team wants to analyze the profitability of products in the portfolio to maximize the value of the portfolio [14]. This is to make decisions on the portfolio, as it is necessary to understand which products are profitable, which are plus/minus zero, and which are non-profitable. A complementary perspective would be to analyze the alignment with business strategy to ensure the strategic fit of products [14]. It would be possible to combine these two perspectives and for example seek to categorize products to green (profitable and strategic), yellow (either profitable, but non-strategic, or non-profitable, but strategic), and red products (neither profitable nor strategic) [17]. Target of analysis in this example is the product portfolio by analyzing individual products. The role involves the PPM team, or a relevant individual, and the information need involves the profitability of sold products. Why the information is needed involves the need to report to the PPM board for PPM decision-making [13].

Figure 2 illustrates the product portfolio management related information needs in accordance with the given example. The product portfolio manager and the subportfolio managers (PPM) need the information on products' profitability and their alignment with strategy.

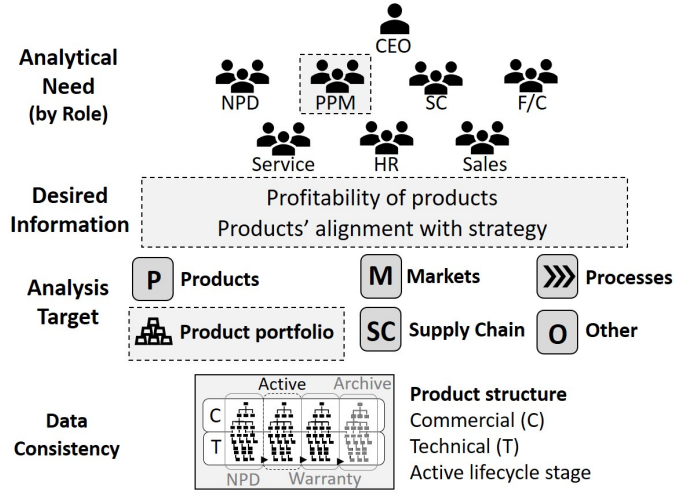

Fig. 2 Information need example - PPM. 
The target of analysis is the entire product portfolio, including all the products. The roles are described as chief executive officer (CEO), new product development (NPD), product portfolio management (PPM), supply chain $(\mathrm{SC})$, finance and control $(\mathrm{F} / \mathrm{C})$, service, human relations (HR), and sales. Nevertheless, the roles can also be described differently. The target of analysis can be individual products, markets, product portfolio, processes, supply chain, or something else (other). In the example the target of analysis is all products, which necessitates the support of product structure, both the commercial and technical [16]. The particular interest is the lifecycle stage where products are sold actively. NPD, warranty, or archive stages are not of interest in this analysis.

\section{Analyzability (II)}

The second step of the concept involves assessing the analyzability and considering whether the desired information is obtainable, and how it is possible to gain. The logic is necessary to be considered while understanding whether existing figures can be used and what the data needs are. It is also necessary to understand how the desired information can be calculated, and whether there are needs to simplify to some extent. The data needs and the suitability of the data, as well as the analysis logic will be considered. The later visualization and presentation of the results depend on the viability of the used data and the applied logic.

Going back to the example of trying to understand product level profitability of all the products in the actively sold product portfolio: Roughly generalizing the profitability of each sold product can be specified by comparing the sales price and the cost of the specific sold product version [9][12]. Only the production costs may then be acknowledged, even though costs accrue through planning and design, manufacturing, delivery, maintenance, and warranty - the total cost of the product [9]. While calculating profitability of individual products there are two options: try to estimate all the costs per product or simplify to some extent. In this example, we choose to simplify and only compare sales price and the direct cost of the product version. The role of product structure is crucial to compare the costs with the sales price in PPM [12][16].

Figure 3 illustrates the logic of analyzing product level profitability according to the discussed example. Sales and cost data are compared for each sold product to reach the product profitability (simplified logic). Each sales item links to a corresponding version item to know exactly which product versions have been provided. This enables linking correct costs to the sold products. The company logic for utilizing enterprise applications may vary, hence the analysis logic must be considered company specifically. Product configurations, sales items, version items, and such can be in different systems, and the definition, or modelling relies on system data standards. The alignment with strategy can be indicated by relevant attributes.

\section{E. Relevant data (III)}

The third step involves the data assets; master data, transactional data, and interactional data relating to products, customers, and other objects of interest [12]. The data assets are necessary to reach factual analysis for reporting and decision-making. The important aspects in considering the relevant data involve the consistency of data, avoiding the challenges of consolidating the data, and the analytics not taking place in individual silos [10]. The needed data link inherently to the previous step of understanding the necessary logic for the analysis, and to the sources of data, likely the enterprise applications [10][11]. These applications inherently relate to business processes [9]. The quality of data assets is crucially essential, however, the traditional approach of relying on enterprise application being responsible for data quality that normally support application-specific transactions [18] may not be sufficient for data quality for digital analytics. This is as there can be different requirements for data and related attributes in the case of analytics [18]. Also, the data quality is the sum of all the steps from capturing the data to analytics and making the decisions [19]. Certain company specifics on data may also be involved [20], affecting the data governance.

The discussed example of product level profitability necessitates identifying the availability and origin of the data relating to sales pricing, sold volumes, technical versions of the sold products, and the related cost information (Figure 3). These are the important data attributes that must be identified, together with their

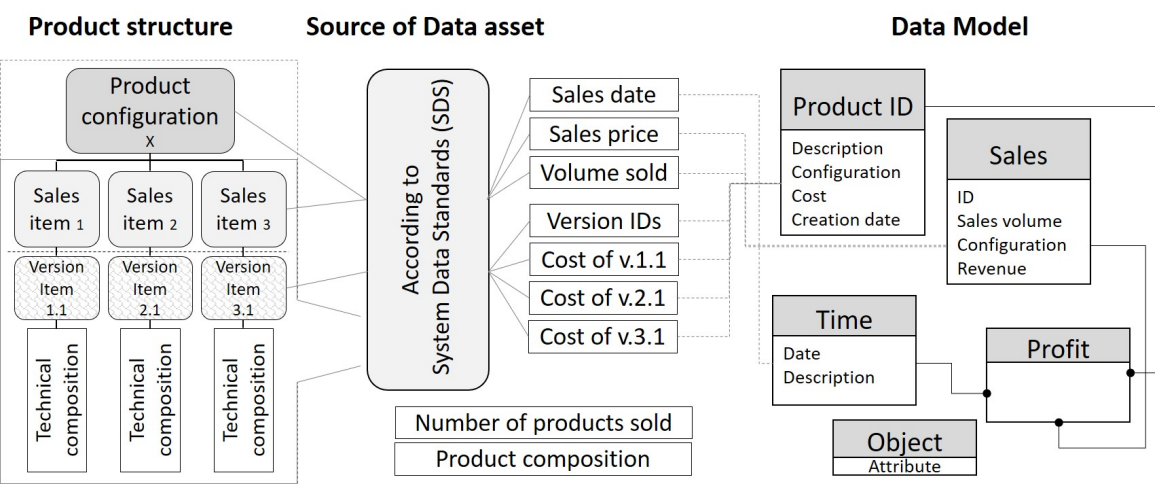

Fig. 3 Analysis logic and data model. 
original source in the enterprise applications. The product structure supports linking of the data in relation to the commercial and technical aspects.

\section{F. Data model (IV)}

The fourth step involves the data model and relates to data domains at a higher level. Data standards is an important aspect of data domains, and can be seen to include business rules, data ownership, usage, data objects and attributes, and their relationships. Data domains can also include item data domain, business partner data domain (suppliers, customers, internal partners, etc.), IoT data domain, transaction data domain, and such. Data domains link also to system data standards, that can be used to define objects and attributes for enterprise applications (PLM, ERP, CRM, etc.). This also enables defining the master application for each attribute. The crucial role of data model for company analytics has been identified [12], but clear focus on how to consider this has been missing. The nature of data assets may also play a factor [11]. Conceptual data model supports understanding which entities can be logically connected with other data entities [21]. The relational database design often bases on conceptual modelling such as UML (Unified Modeling Language) [22]. Products and related attributes can be addressed by a conceptual data model as object and attributes (Figure 3), for example a product with a certain product-ID [23]. Conceptual data model can then be linked to a logical database, and the implementation of the data model is enabled by generated physical data model [23]. Different technological arrangements exist and their application for the intended purpose might be possible without significant costs [15]. Data model, however, needs to be considered at a higher level and separate from technology.

In the discussed example, the cost of a specific product ID can be assessed by the support of data model by linking the relevant objects and attributes. The relevant attributes in the example would be the product description, specific configuration that was sold, and the cost. This is compared with the sales that has relevant attributes, including the ID, volumes, configuration, and the revenue. This allows to structure relevant data by the means of a conceptual data model by acknowledging the necessary relationships.

\section{G. Visualization $(V)$}

The fifth step, visualization, involves the analysis and reporting necessary for decision-making. The information needs and the analyzed aspects that relate to the specific target of analysis can be visualized to a meaningful form to enable decision-making based on factual information. The visualization can form a level of management dashboard, but importantly should reflect the user needs and goals [24]. The visualization links inherently to the technologies for the analysis and handling of data. The application of existing technologies might be possible
[15]. For example, the application of cloud data platforms to capture and store data in the cloud to improve computational efficiency [25] to form a spider web type model for the flexible combination of data. The US military cloud computing efforts is a related example. The technological considerations include the axis of raw data data ingestion - data process to extract the most valuable insights and benefits from the data [26]. One of the keys is to avoid the challenges caused by legacy systems.

In the context of the discussed example, the data visualization for beneficial insights in terms of product level profitability, and knowing which products are strategic, would enable addressing one aspect of product portfolio related analysis and decision-making [9]-[16]. However, the application is not limited here, but similarly analytics on supply chain, markets, and such can prove beneficial for businesses.

\section{DISCUSSION}

Obtaining business relevant information from data to gain valuable insights to support decision-making can provide a competitive edge for companies. It is known how digital technologies and data can provide opportunities; however, the challenge is yet to be supported by effective concepts. Digitizing the company analytics to enable more systematic exploitation of data and to avoid old more manual analysis and challenges, necessitates understanding the needed foundations, company information needs, logic necessary for the analytics, relevant data, and data model, including data domains that cover relevant objects and attributes. Finally, the obtained analysis results need to be possible to visualize to gain the valuable insights.

The developed concept was validated initially. Industry experts saw the model to be extremely timely and relevant. The content of the data model step was discussed the most, and the term data domain was introduced to link with relevant industry terms. Comments mostly involved the levels of terminology. The model was seen as realistic. The necessary technology was stated to require company specific consideration.

The needed technologies to apply the concept already exist. Product portfolio management was used as an example while discussing the concept. However, the concept can be applied to a variety of information needs, involving products, markets, product portfolio, processes, and the supply chain. Variety of information needs can be satisfied by the effective, digital application of data assets.

The analytics might be possible to arrange also within individual IT applications in some instances but might be affected by issues caused by siloed company structures, system integration, or data consistency. In this concept, the data consistency is ensured by the consistent logic involving productization and product structure. The discussed example relates to product portfolio management analytics where the data is linked to products and the corresponding structure, with an aim to understand product level profitability and the strategic 
nature of products. Further visualizing of customers might also be possible through products by considering strategic aspects, profit, value, or other vital issues. Similarly supply chain visualization would be possible through products or smaller related structural elements. The opportunities could relate, for example, to supply chain optimization, procurement, and analyzing sales channels. Similarly, field data might provide opportunities through IoT data and digital footprint. Similar opportunities exist for sales, after sales, or even recycling components, all through the products and the related structure. Hence, this study reveals the need for digital product portfolio management by connecting business performance, and products through their lifecycle. Similar digital analytics are possible to serve the related motivations. Effectively, the application of the presented concept would digitalize the entire company ecosystem.

Finding: A path from information needs to visualizing company analytics necessitates considering the analysis needs, analysis logic, relevant data, necessary data model, and the necessary technologies. Company business processes, IT applications, and data assets provide the foundations for digital business analytics.

\section{REFERENCES}

[1] B. Tabrizi, E. Lam, K. Girard, and V. Irvin, "Digital transformation is not about technology", Harv. Bus. Rev. 13 March, 2019.

[2] C. Dremel, E. Stoeckli, J. and Wulf, "Management of analytics-as-a-service - results from an action design research project," J. Bus. Analytics, vol. 3, no. 1, pp. 1-16, 2020.

[3] C. Dremel, M. Herterich, J. Wulf, J.-C. Waizmann, and W. Brenner, W. "How AUDI AG Established Big Data Analytics in Its Digital Transformation," MIS Q. Exec., vol. 16, no. 2, pp. 81-100, 2017.

[4] C. Gröger, "Building an Industry 4.0 Analytics Platform," Datenbank Spektrum, vol. 18, pp. 5-14, 2018.

[5] S. Groggert, M. Wenking, R. H. Schmitt, and T. Friedli, "Status Quo and Future Potential of Manufacturing Data Analytics - An Empirical Study," in Proc. IEEE Int. Conf. Indust. Eng. Manag., IEEM, Singapore, pp. 779-783, 2017.

[6] R. Wdowik, and R. M. C. Ratnayake, "Collaborative Technological Process Planning with 5G Mobile Networks and Digital Tools: Manufacturing Environments' Perspective," in Proc. IEEE Int. Conf. Indust. Eng. Manag., IEEM, Macao, pp. 349-353, 2019.

[7] E. Govender, A. Telukdarie, and M. N. Sishi, "Approach for Implementing Industry 4.0 Framework in the Steel Industry," in Proc. IEEE Int. Conf. Indust. Eng. Manag., IEEM, Macao, pp. 1314-1318, 2019.

[8] J. Echterfeld, and J. Gausmeier, "Digitising Product Portfolios," Int. J. Innovation Manag., vol. 22, no. 5, article $1840003,2018$.

[9] H. Hannila, A. Tolonen, J. Harkonen, and H. Haapasalo, "Product and supply chain related data, processes and information systems for product portfolio management", Int. J. Prod. Lifecycle Manag., vol. 12, no. 1, pp. 1-19, 2019.

[10] J. Harkonen, E. Mustonen, and H. Hannila H. "Productization and Product Structure as the Backbone for
Product Data and Fact-Based Analysis of Company Products", in Proc. IEEE Int. Conf. Indust. Eng. Manag., IEEM, Macao, pp. 474-478, 2019.

[11] H. Hannila, R. Silvola, J. Harkonen, and H. Haapasalo, "Data-driven begins with DATA; potential of data assets", J. Computer Inf. Systems, DOI: 10.1080/08874417.2019.1683782, 2019.

[12] H. Hannila, J. Koskinen, J. Harkonen, and H. Haapasalo, "Product-level profitability - Current challenges and preconditions for data-driven, fact-based Product Portfolio Management", J. Enterprise Inf. Manag., vol. 33, no. 1, pp. 214-237, 2020.

[13] A. Tolonen, J. Harkonen, and H. Haapasalo, "Product Portfolio Management - Governance for Commercial and Technical Portfolios Over Life Cycle", Technol. Invest. vol. 5, no.4, pp. 173-183, 2014.

[14] A. Tolonen, M. Shahmarichatghieh, J. Harkonen, and H. Haapasalo, H. "Product Portfolio Management - Targets and Key Performance Indicators for Product Portfolio Renewal over Life Cycle", Int. J. Prod. Econ., vol. 170, pp. 468-477, 2015.

[15] J. Koskinen, E. Mustonen, J. Harkonen, and H. Haapasalo, "Product-level profitability analysis and decision-making: Opportunities of IT application-based approach", Int. J. Prod. Lifecycle Manag., in press.

[16] N. Lahtinen, E. Mustonen, and J. Harkonen, J. "Commercial and technical productization for fact-based product portfolio management over life-cycle", IEEE Transactions Eng. Manag., DOI: 10.1109/TEM.2019.2932974, 2020.

[17] A. Tolonen, J. Harkonen, M. Verkasalo, and H. Haapasalo, "Product Portfolio Management Process over Horizontal and Vertical Portfolios", Int. J. Prod. Lifecycle Management, vol. 8, no. 3. pp. 189-215, 2015.

[18] A. McAfee and E. Brynjolfsson E. "Big data: the management revolution", Harv. Bus. Rev., vol. 90, pp. 6168, 2012.

[19] V. C. Storey, R. M. Dewan, and M. Freimer, "Data quality: setting organizational policies" Dec. Supp. Syst., vol. 54, pp. 434-42, 2012.

[20] J. Stark, PLM and Product Data: Product Lifecycle Management, vol. 1, pp 159-219, 2019.

[21] V. C. Storey, and I.-Y. Song, "Big data technologies and Management: What conceptual modeling can do", Data \& Knowl. Eng., vol. 108, pp. 50-67, 2020.

[22] L. Li, and X. Zhao, "UML specification and relational database", J. Object Technol., vol. 2, no. 5, pp. 87-100, 2003.

[23] A. de la Vega, D. García-Saiz, C. Blanco, M. Zorrilla, and P. Sánchez, "Mortadelo: Automatic generation of NoSQL stores from platform-independent data models", Future Generation Comput. Systems, vol. 105, pp. 455-474, 2020.

[24] A. Sarikaya, M. Correll, L. Bartram, M. Tory, and D. Fisher, "What Do We Talk About When We Talk About Dashboards?", IEEE Transactions Visualization Comput. Graphics, vol. 25, no. 1, pp. 682-692, 2019.

[25] K.-F. Ho, H. W. Hirai, H.W, Y.-H. Kuo, H. M. Meng, and K. K. F. Tsoi, "Indoor Air Monitoring Platform and Personal Health Reporting System: Big Data Analytics for Public Health Research", IEEE Int. Congress Big Data, pp. 309-312, 2015.

[26] A. Faroukhi, I. El Alaoui, Y. Gahi, and A. Amine, "Big data monetization throughout Big Data Value Chain: a comprehensive review", J. Big Data, vol. 7, no.1, artic. 3, 2020 . 